\title{
ECG SIGNAL CLASSIFICATION BASED ON DEEP LEARNING BY USING CONVOLUTIONAL NEURAL NETWORK (CNN)
}

\author{
Aqeel M.Hamad alhussainy ${ }^{1}$, Ammar D. Jasim² \\ ${ }^{1,2}$ College of Information Engineering, Al-Nahrain University, Baghdad, Iraq \\ aqeel-alhussainy@utq.edu.iq ${ }^{1}$, ammar@coie-nahrain.edu.iq ${ }^{2}$ \\ Received:17/3/2020, Accepted:26/06/2020
}

\begin{abstract}
Cardiovascular Diseases (CVDs) are consider main cause of death today a according to World Health Organization (WHO). Because ECG signal is very important tool in monitoring and diagnosis of these disease , different automatic methods are proposed based on this signal. The manual analysis of ECG signals is suffered different challenges such as differeculty of detecting and classify waveform of this signal, So, many machine learning methods are explored to describe the anomalies ECG signal accurately . Deep learning (DL) can be used in ECG classification, it can improve the quality of the automatic classification system. In this paper, We have proposed a deep learning classification system using different layers of convolution, rectifier and pooling operations that can be used to increase feature extraction of ECG signal. We have proposed two models, one is used 1-D signal, in which we designed model for classification csv type of data for ECG signal, while in the second proposed system, we used model for 2-D signal after convert it from its csv type . 2-D signal (ECG image) is used in order to augment the two dimensional signal with different methods to increase the accuracy of the model by training it with geometric transformation of the original input images such as rotation, shearing etc. The results are compared with AlexNet and other models based on different metrics, which are used to measure performance of proposed work, the result show that, proposed models improves efficiency of the classification in the two systems.
\end{abstract}

\section{INTRODUCTION}

Electrocardiogram (ECG) is very important tool in diagnosis of heart disease. It is a waveform used to explain the activity of heart, any up normality in this signal is an indication of heart problem, so this signal can be used in heart disease analysis[2]. Different ECG classification techniques were proposed to avoid the manual analysis of ECG signal problems, also different machine learning methods were used to detect the up normality of this signal. The machine methods are constructed from features mapped such as wavelet transform, stochastic method and classification such as decision tree and Bayesian classification, the mapped or extracted features represent the properties and characteristics of the signal, while classification is used to analysis and classify these features [3],[4].

In recent years, Deep learning, has used for data analysis. It is used for big data processing and other applications such as image classification, speech recognition and more applications, which are require complicated processing [5]. In this paper, we have proposed anew Convolutional Neural Network (CNN), to classify ECG signals. We have made several contributions by design a new model as well as applying different augmentation methods to increase accuracy of the system. We have proposed two CNNs model, the first model is one dimension model, which is used one dimension signal (CSV dataset), this model is constructed from different types of 1-D layers such as convolution, ReLu, add and Max pooling layer. While the second model is two dimensional model, which is used two dimension signal (image) as input, this image is obtained by drawing 1-D signal and save it as image to be used for training and testing the model, which is constructed from different 2-D layer such as convolution, max. pooling and soft layer. Two dimension model is very important because it is used data augmentation, which increase the accuracy of the classification. The proposed methods 
are trained and tested by using MIT-BIH dataset.The performance of the proposed methods are evaluated by using different metrics such as accuracy, precession, recall and F score.

\section{Literatue SURVEY}

Ankit Sanghvi and Sachin M. Bojewar in (2014) proposed an ECG classification model based on extraction feature of ECG image by using wavelet transform to remove the high frequency noise, they are exploited wavelet transform to convert the signal into different sub band with different resolution, which can be used to analysis the ECG signal [6].

Stalin Subbiah, Rajkumar Patro and Subbuthai in (2015), they are proposed a classification system by prepressing the input signal to remove the signal noise, in which, there are different filters used such as FIR filter to remove the noise from , also they used (BPN) and (SVM) as classification methods [7].

Zhaohan Xiong et.al proposed In (2017) CNN system for ECG signal classification. They used 16-layer 1D Convolutional Neural Network (CNN). Also they used batch normalization to normalize the layers, down sampling is used by adding pooling layer which represented by max. pooling or average pooling, the data that is used was 8528 for training while for testing they used 3658 [5].

C. Venkatesani , P. Karthigai, Anand, S. Satheeskum and R. Kumars in (2018) proposed a system to detect the up normality of ECG signal by prepressing the input ECG signal with DENLMS method, which is used with other filter to get high quality with less overhead. DWT was used for approximation coefficients extraction, then QRS complex is recognized then they used frequency domain features which is classified by using SVM classification to detect up normality [1].

In (2018), Mohammad Kachuee et.al proposed ECG classification technique by using transferable approach. The proposed work was constructed from five layers with pooling and rectifier unit as activation function[8] . Tae Joon Jun and Hoang Minh Nguyen in (2018) used 2-D CNN for ECG classification, where the 2D signal was image of ECG after convert it into image.They used 2D ECG to classify it into normal or to seven arrhythmia. The classification system was made with deep learning CNN model with (11) layers that is constructed from convolutional layers with activation and pooling layers[9].

Most of related work are used basic features of the input ECG signal after extract it depending on different methods such as global features or by using frequency transformation techniques such as wavelet transform and discrete cosine transform, these features are used as input to classifier method such as Support Vector machine (SVM),Artificial Neural Network (ANN), unfortunately these feature may not represent the original signal effectively especially when the signal is suffered from any type of noise ,which make the extracted feature not effective, also some deep leaning method is used one dimension model, the problem with one dimension signal is that the dataset cannot be augmented, data augmentation is very necessary especially when the dataset is not balanced,(i.e the different classes have different numbers of labels), In this paper, we have proposed anew Convolutional Neural Network (CNN), to classify ECG signals. We have made 
several contributions by design a new model as well as applying different augmentation methods to increase accuracy of the system. We have proposed two CNNs model, the first model is one dimension model, which is used one dimension signal (CSV dataset), While the second model is two dimensional model, which is used two dimension signal (image) as input, this image is obtained by drawing 1-D signal and save it as image to be used for training and testing the model, which is constructed from different 2-D layer such as convolution, max. pooling and soft layer. Two dimension model is very important because it is used data augmentation, which increase the accuracy of the classification by increasing the balance of the dataset. The proposed methods are trained and tested by using MIT-BIH .

\section{Methodology}

In this paper, we have proposed two deep learning algorithms to classify ECG input data, the first system was based on 1-D CNN model, which is accepted the input data as csv data, while the second system was based on 2-D input signal(image).

\section{A. One dimension proposed system}

This system is constructed from the following stages:

1) Data collection: The dataset is constructed from the PTB Diagnostic ECG Database. and the MIT-BIH Arrhythmia Dataset. The number of samples in both collections are used for training a deep neural network. Table I describes the details of each category of dataset.

TABLE I

CATEgory OF THE MIT-BIH ECG Dataset
\begin{tabular}{|c|c|}
\hline Class & Condition \\
\hline N & Normal \\
\hline S & Supra-ventricular premature \\
\hline V & Ventricular escape \\
\hline F & Normal and ventricular (Fusion) \\
\hline Q & Paced \\
\hline
\end{tabular}

2) Prepressing: This stage is used to arrange the number of samples for each ECG signal which must be equal for all the record, also the dataset is divided into training and test data.

3) Design, training and testing 1-D model: We have design a new CNN model that is constructed from the layers ,which is shown in Fig. 1. The input is csv data (one dimension data),this input is passed through sequential layers of convolution, rectified linear unit layer(ReLu), max.pooling, add, fully and dense layers with the sequence that is shown in Fig. 1, which is described the details of the proposed model.At first, the input data is added with the result of first three layers (conv-1,relu-1and conv-2) to increase the extracted features, then this features are filtered by another Relu layer $(\operatorname{ReLu} 2)$, this combination of layers is repeated five times, then other layers are used to arrange the data and convert it into five class by soft max and classification layers.

4) Calculate the performance metrics: There are three basic metrics must be calculated to determine the efficiency of the method, all these metrics are evaluated by determine the following detections:

a) (TP) : true detect arrhythmia. 


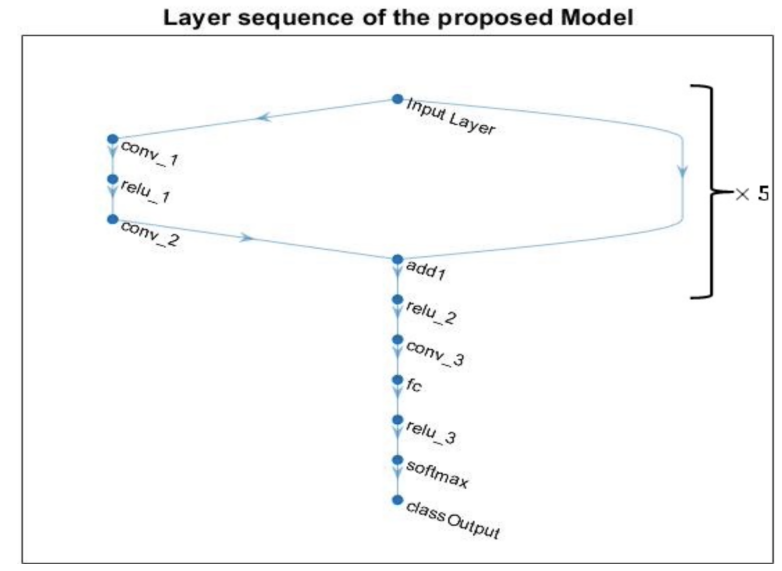

Figure 1: The proposed CNN Model architecture

b) $(\mathrm{TN})$ : true normally detected.

c) (FP): false detected as positive.

d) $(\mathrm{FN})$ : false detected as normal.

Accuracy: Represent ratio between the result that is correctly predicted (positive and negative) to the sum of all predictions.[12],[13]

$$
A c c=\frac{T N+T P}{F P+F N+T P+T N}
$$

Precision: Represent the ratio of the correctly positive to the total positive perdition .[12],[14]

$$
A c c=\frac{T P}{F P+T P}
$$

Recall: Represent the ratio between the identify positive to the all truly positive( true positive and false negative)[12],[14]

$$
A c c=\frac{T P}{T P+F N}
$$

F1-score: This factor represent normalize average of recall and precision.[14,15]

$$
\text { Acc }=\frac{2 * \text { Precision } * \text { recall }}{\text { Precision }+ \text { recall }}
$$

\section{B. Two dimension proposed classifier system}

The second proposed system is used 2-D signal (image) in the input stage, this signal can be constructed by converting 1-D signal (csv data) into image. Two dimension signal is very important to be used in augmentation of data, which cannot be performed in one dimensional ECG signal because the distortion of signal, the proposed classification method are 
described by the following steps:

- Read input data The dataset consists of (CSV) files, each row represents a signal in the dataset. The final element of each row determines the class of the signal. MIT.BIH arrhythmia data is used in our paper.

- Data preprocessing The proposed CNN model requires 2D data , so We must first convert the (csv) data to 2D signal( images), which is accomplished by design function that read data, plot the desired image, resize it to be used as input to the desired model and save the image. Two dimension signal is very important in data augmentation because the original signal is very hard to be augmented.

- Data Augmentation In one dimension model, it is very hard to add data augmentation in training set because the distortion of one dimension signal. Two dimension augmentation with different method can increase the accuracy of the model by provide different viewpoints of the same signal, this property is very important especially in medical application because the most of dataset are normal and the dataset is unbalanced. There are different methods of augmentation such as rotation ,shearing, cropping etc. In this paper, we used different cropping methods such as left, right, top and bottom method in order to provide different shapes for the same image and increase model accuracy.

- Design CNN model We have proposed (2-D CNN) classifier for ECG beats signals. This model is constructed from convolutions, pooling and dense layer in addition to other inherent layers like rectifier layer, which is used as activation function after convolution layer, in this paper we used (ReLU) as activation function.

$$
R_{e} L U(x)=\max (0, x)
$$

In order to reduce the amount of the overfitting, batch normalization is used, which can make the network more stable, this is accomplished by discard the mean batch and normalized it by using standard deviation as described in Equation 6 and Equation 7 respectively.

$$
\begin{gathered}
\partial 2 B=\sum_{\infty}^{n=1}\left(x_{i}-\mu B\right)^{2} \\
x_{i}=\frac{\left(x_{i}-\mu B\right)}{\sqrt{\partial 2 B}+\epsilon}
\end{gathered}
$$

The convolution layer convolve the layers output with filters by dot product them according to specified size of window, in this model, We are use convolution layer with window size $(3 * 3)$, while pooling layer is used to down sampling the output by chose the maximum number for each $(2 * 2)$ window. The proposed CNN model is described in Fig. 2. As shown in figure, three convolution layers with 64 filter are applied sequentially, then max. pooling method with pool size ( $2 * 2)$ is used to reduce the size of the data, then two convolution layers with filter 128 is used followed by max. pooling layer $(2 * 2)$, then another convolution layer with 256 filter is used to extract the features details, third max. pooling layer is used 
to reduce the data to lowest volume, then fully,dens5 and soft max layers are used to classify the data into five categories based on the extracted characteristics.

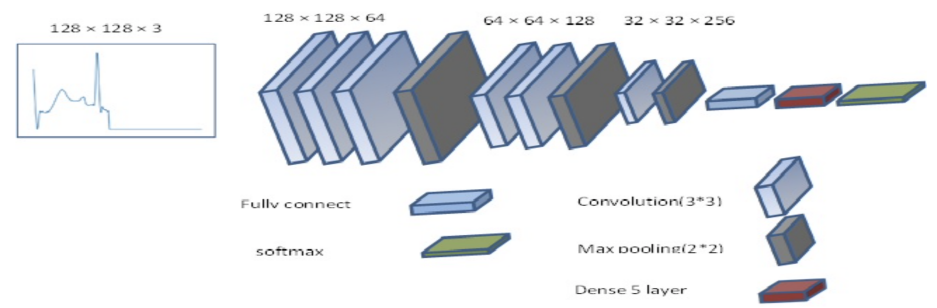

Figure 2: The structure of the suggested 2D CNN model

\section{Experimental Results AND Discussion}

The proposed models are trained and tested by using MIT-BIH ECG dataset, which is divided into train set and test set. The experiments are performed on core i7 2.9GHz CPU, 16G.B RAM by using matlab 2019. We have used Stochastic Gradient Decedent (SGD) with momentum for leaning the model, also the training of the model was based on different batch size but the best patch size was 256 , learning rate $=0.001$ and weight decay was used to overcome over fitting problem, also the experiments are performed by using 80 epochs for training the first model(1-D model) and 20 epochs for training the second model (2-D model). The performance of the proposed methods are evaluated by using different metrics such as accuracy, precession, recall and F score, the results are compared with another paper as described in the following sections.

\section{A. Results of 1-D Model}

We have proposed one dimension model by design CNN model to increase the accuracy of classification of heart disease , this model is trained and compared with other approaches. A first, the MIN-BIH arrhythmia ECG dataset is divided into training and test sets, the size of the training set was(109150) samples, while the size of the testing set was (4000)in which, we are take random data for each category to be used as testing data. Fig. 3 shows the relations between training and validation accuracy and loss plots verses the epochs iterations, from figure, there is small amount of over fitting that is decreased with increasing the numbers of iterations. Also the accuracy on validation data is improved with increasing epochs iteration as shown in Fig. 3.The accuracy reached to $99.97 \%$ in the training of the model, while in the testing, the accuracy was $95.7 \%$, average precision : 0.977 and ranking loss : 0.013 .
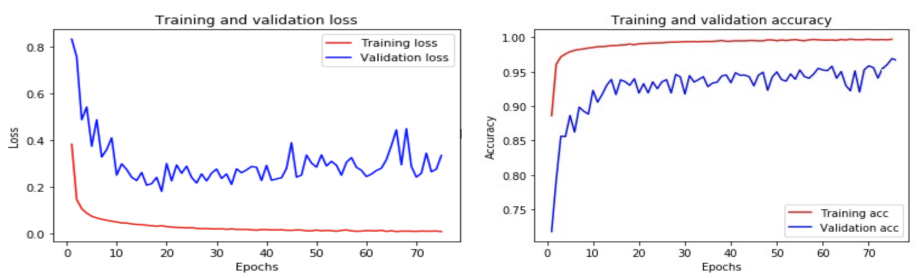

Figure 3: The relations between training and validation accuracy and loss 
TABLE III

Proposed Algorithm With Different Filters VAlues

\begin{tabular}{|c|c|c|c|c|c|c|c|c|c|}
\hline Category & \multicolumn{3}{|c|}{16 filters } & \multicolumn{3}{c|}{ 32 filters } & \multicolumn{3}{c|}{ 64 filters } \\
\hline & precision & recall & f1-score & precision & recall & f1-score & precision & recall & f1-score \\
\hline 0 & 0.77 & 1.00 & 0.87 & 0.92 & 0.99 & 0.92 & 0.85 & 1.00 & 0.92 \\
\hline 1 & 0.99 & 0.77 & 0.87 & 0.98 & 0.87 & 0.92 & 1.00 & 0.88 & 0.93 \\
\hline 2 & 0.94 & 0.95 & 0.95 & 0.97 & 0.96 & 0.96 & 0.95 & 0.96 & 0.96 \\
\hline 3 & 0.98 & 0.92 & 0.95 & 0.99 & 0.97 & 0.98 & 0.98 & 0.93 & 0.96 \\
\hline 4 & 1.00 & 0.98 & 0.98 & 1.00 & 0.99 & 0.99 & 1.00 & 0.99 & 0.99 \\
\hline micro avg & 0.92 & 0.92 & 0.92 & 0.96 & 0.96 & 0.96 & 0.95 & 0.95 & 0.95 \\
\hline macro avg & 0.94 & 0.92 & 0.92 & 0.96 & 0.96 & 0.96 & 0.96 & 0.95 & 0.95 \\
\hline weighted avg & 0.94 & 0.92 & 0.92 & 0.96 & 0.96 & 0.96 & 0.96 & 0.95 & 0.95 \\
\hline
\end{tabular}

TABLE IV

Performance Metrics For Each Class

\begin{tabular}{|c|c|c|c|}
\hline Classes & Accuracy $\%$ & Precision $\%$ & f1-score \\
\hline $\mathrm{N}$ & 98.51 & 0.92 & 0.92 \\
\hline $\mathrm{S}$ & 92.86 & 0.98 & 0.92 \\
\hline $\mathrm{V}$ & 96.62 & 0.97 & 0.96 \\
\hline $\mathrm{F}$ & 91.15 & 0.99 & 0.98 \\
\hline $\mathrm{Q}$ & 99.37 & 1.00 & 0.99 \\
\hline
\end{tabular}

The performance of the system is measured by the evaluation metric as shown in Table II, the proposed system is achieved better results in term of precision, which is $96 \%$,with accuracy value is $97.7 \%$ and recall $=96 \%$, which is good improvement as compared with others two approaches shown in Table II. The number of filters have a great influence on the model results ,Table III describes performance specification for the proposed model with different filters (16,32 and 64), from table, the best result for the system is satisfied with 32 filter for convolution layers, while the performance metrics for each class is shown in Table IV.

TABLE II

Performance Metrics For The Proposed Algorithm Compared With Others Approaches

\begin{tabular}{|c|c|c|c|}
\hline Method & Precision & Recall & accuracy \\
\hline Ref[16] & 0.928 & 0.937 & 0.955 \\
\hline Ref[1] & 0.95 & 0.95 & 0.951 \\
\hline Proposed algorithm & 0.96 & 0.96 & 0.957 \\
\hline
\end{tabular}

The confusion matrix of results is shown in Table V, We note that, there is high improvement in the matching between the predicting results and the actual testing data. The diagonal diameter describes the matching values, however there is low range of error, which is appeared in the matrix as compare with other approaches .

\section{B. Results of 2-D Model}

In this paper, an enhanced two dimension convolutional network system and algorithm are proposed for prediction of heart disease and classify it if its is founded. The system is started by converting one dimensional input data into two dimension signal (image) for ECG signal of dataset, then this data is augmented as shown in Fig. 4, which describes the original image and different views for the same image by using different cropping methods, then these data are used as input to the proposed 2-D CNN model for training and classification. 


\begin{tabular}{|c|c|c|c|c|c|c|}
\hline & \multicolumn{6}{|c|}{ Confusion matrix without normalization } \\
\hline \multirow{5}{*}{ True label } & $\mathrm{N}$ & 797 & 2 & 10 & 0 & 0 \\
\cline { 2 - 7 } & $\mathrm{S}$ & 90 & 684 & 4 & 3 & 0 \\
\cline { 2 - 7 } & $\mathrm{V}$ & 20 & 3 & 773 & 3 & 1 \\
\cline { 2 - 6 } & $\mathrm{F}$ & 25 & 0 & 43 & 732 & 0 \\
\cline { 2 - 6 } & $\mathrm{Q}$ & 5 & 0 & 0 & 0 & 795 \\
\cline { 2 - 6 } & & $\mathrm{N}$ & $\mathrm{S}$ & $\mathrm{V}$ & $\mathrm{F}$ & $\mathrm{Q}$ \\
\cline { 2 - 5 } & \multicolumn{7}{|c|}{ Predicted Label } \\
\hline
\end{tabular}
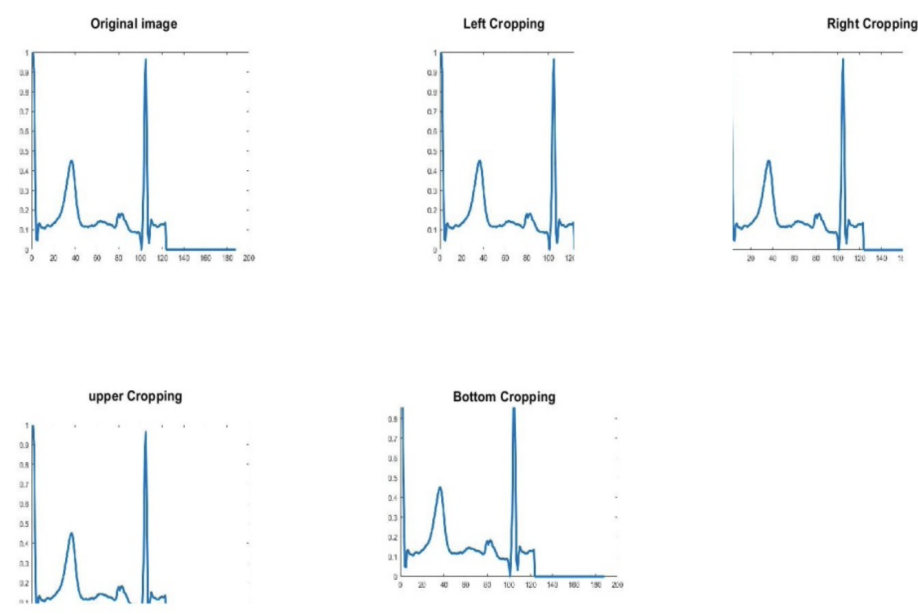

Figure 4: Data augmentation with different cropping methods

The original image of different ECG beats are shown in Fig. 5 , which can be augmented by using cropping methods as shown in Fig. 4, the augmented ECG image can increase the different view of the image and increase the accuracy of the classifier. The data set was 1250 which are collected by draw the one dimension signal of ECG, these images are distributed evenly across five classes, they are augmented by using four different cropping methods(left bottom cropping, left upper cropping, right bottom cropping and right upper cropping), so the total image were 6250 image, 5000 images are dedicated for training phase, while the remainder images(1250) are used for testing stage. The proposed model is trained for 20 epochs as shown in Fig. 6 , which describes the loss function verses epoch training iterations. It is clear that, there is small over fitting between training and validating loss, which is decreased with increasing the number of iteration epochs . 
TABle Vi
Performance Comparison Of The Proposed Algorithm Verses Alexnet Model And Ref[9]

\begin{tabular}{|c|c|c|c|c|c|}
\hline Method & Data set & Precision & Recall & F1-score & accuracy \\
\hline \multirow{2}{*}{ AlexNet model } & Native Data & 82.67 & 84.496 & 80.77 & 80.40 \\
\cline { 2 - 6 } & With augmentation & 91.893 & 91.47 & 91.624 & 91.27 \\
\hline \multirow{2}{*}{ Ref [9] method } & Native Data & 81.471 & 81.02 & 81.072 & 81.2 \\
\cline { 2 - 6 } & With augmentation & 88.15 & 85 & 85.53 & 85.27 \\
\hline \multirow{2}{*}{ Proposed model } & Native Data & 87 & 81 & 82 & 84 \\
\cline { 2 - 6 } & With augmentation & 92.325 & 92.639 & 91.992 & 91.93 \\
\hline
\end{tabular}

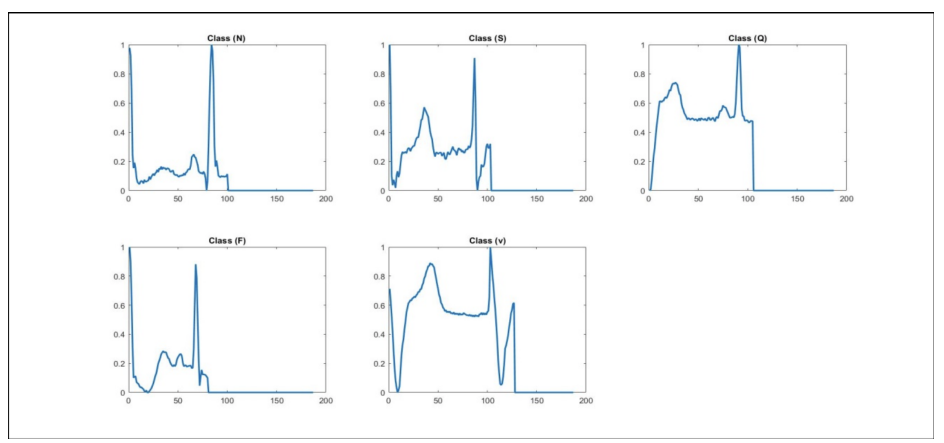

Figure 5: The original images of five classes of ECG images

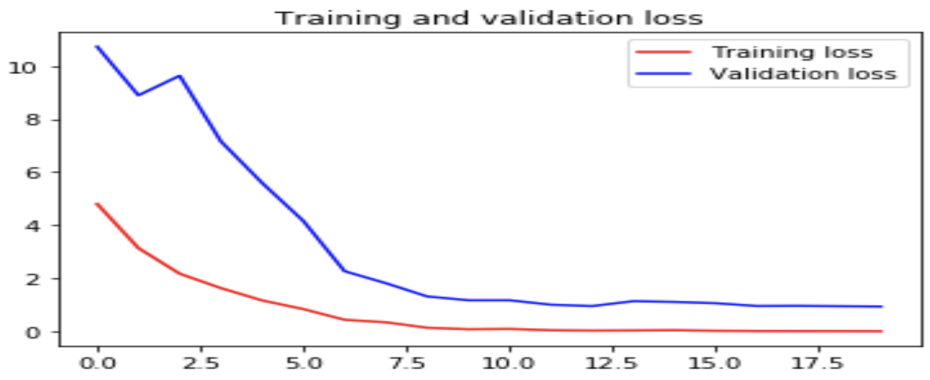

Figure 6: Training and validation loss

To measure the efficiency of the proposed system, we compare it with other classification work. Our system is achieved high improvement as compared to other classification model. Table VII shows the advance improvement in result of the proposed system as compared with Alex net and Joon approach [9], in terms of precision, recall, F score and accuracy.

Table VII shows the results of Alexnet for the five classes of ECG classification, while Table VIII describe the correlation between the actual and predicted values for the different classes by using the proposed system, from Tables VII and VIII We are noted that, although (TP) is less in the proposed method than Alexnet, the proposed method is better because it has the lowest values for (FP).

In Tables IX and X, We are observed the difference in the values of compatibility between prediction and actual for the 
TABLE VII

Confusion Matrix For Alexnet Model Without Augmentation

\begin{tabular}{|c|c|c|c|c|c|c|}
\hline \multicolumn{2}{|c|}{ Classes } & \multicolumn{5}{|c|}{ Actual } \\
\cline { 3 - 8 } \multicolumn{1}{|c|}{} & N & S & V & F & Q \\
\hline \multirow{4}{*}{ Prediction } & N & 50 & 2 & 0 & 0 & 0 \\
\cline { 2 - 8 } & S & 10 & 42 & 0 & 0 & 0 \\
\cline { 2 - 8 } & V & 6 & 0 & 38 & 0 & 0 \\
\cline { 2 - 8 } & F & 13 & 1 & 0 & 34 & 1 \\
\cline { 2 - 8 } & Q & 6 & 0 & 0 & 1 & 46 \\
\hline
\end{tabular}

TABLE VIII

Confusion Matrix For The Proposed Classification System Without Augmentation

\begin{tabular}{|c|c|c|c|c|c|c|}
\hline \multicolumn{2}{|c|}{ Classes } & \multicolumn{5}{|c|}{ Actual } \\
\cline { 2 - 7 } \multicolumn{1}{|c|}{} & N & S & V & F & Q \\
\hline \multirow{4}{*}{ Prediction } & N & 44 & 0 & 0 & 2 & 0 \\
\cline { 2 - 7 } & S & 4 & 48 & 0 & 1 & 0 \\
\cline { 2 - 7 } & V & 5 & 0 & 47 & 1 & 0 \\
\cline { 2 - 7 } & F & 10 & 0 & 0 & 33 & 0 \\
\cline { 2 - 7 } & Q & 13 & 1 & 0 & 10 & 31 \\
\hline
\end{tabular}

proposed method. There is a significant evolution in the values of (TP) in the proposed method as compared with Alexnet, which is leaded to good results as shown in the tables.

TABLE IX

Confusion Matrix For Alexnet Model For Augmented Data

\begin{tabular}{|c|c|c|c|c|c|c|}
\hline \multirow{2}{*}{ Classes } & \multicolumn{5}{|c|}{ Actual } \\
\cline { 3 - 8 } & & $\mathrm{N}$ & $\mathrm{S}$ & $\mathrm{V}$ & $\mathrm{F}$ & $\mathrm{Q}$ \\
\hline \multirow{5}{*}{ Prediction } & $\mathrm{N}$ & 48 & 2 & 2 & 29 & 5 \\
\cline { 2 - 8 } & $\mathrm{S}$ & 7 & 236 & 0 & 27 & 4 \\
\cline { 2 - 7 } & $\mathrm{V}$ & 0 & 0 & 286 & 5 & 1 \\
\cline { 2 - 7 } & $\mathrm{F}$ & 8 & 0 & 0 & 380 & 3 \\
\cline { 2 - 7 } & $\mathrm{Q}$ & 5 & 6 & 2 & 20 & 325 \\
\hline
\end{tabular}

TABLE $X$

Confusion Matrix Of Proposed Model For Augmented Data

\begin{tabular}{|c|c|c|c|c|c|c|}
\hline \multirow{2}{*}{ Classes } & \multicolumn{5}{|c|}{ Actual } \\
\cline { 3 - 8 } & & $\mathrm{N}$ & $\mathrm{S}$ & $\mathrm{V}$ & $\mathrm{F}$ & $\mathrm{Q}$ \\
\hline \multirow{5}{*}{ Prediction } & $\mathrm{N}$ & 54 & 7 & 0 & 9 & 7 \\
\cline { 2 - 8 } & $\mathrm{S}$ & 4 & 277 & 0 & 2 & 6 \\
\cline { 2 - 8 } & $\mathrm{V}$ & 3 & 5 & 364 & 2 & 0 \\
\cline { 2 - 8 } & $\mathrm{F}$ & 18 & 23 & 0 & 327 & 5 \\
\cline { 2 - 8 } & $\mathrm{Q}$ & 10 & 12 & 2 & 6 & 357 \\
\hline
\end{tabular}




\section{Conclusion}

In this paper, two types of classification and prediction system were proposed based on deep neural network by design convolutional neural network to increase the heart disease prediction and classification accuracy . The first system is based on one dimension input signal, which is used the csv data a input. We are designed CNN model based on convolution, pooling and addition layer with other non-linearity functions such as ReLu, regularization. The input data was MIT-BIH ECG dataset, which have (187) samples for each ECG beat signal, these input signal s are convolved and trained with the proposed system and then tested with other part of the dataset, which is selected randomly to measure the proposed system efficiency. The proposed system is achieved accuracy better than other systems according to the performance metrics that are used to evaluate the model accuracy. The accuracy was $97.7 \%$, precision $=96 \%$,recall $=96 \%$, which are better than other systems as described in Table II . The second system is used two dimension signal. At first, the input signal (.csv data) is converted into two dimension signal by plot the ECG image from the original data and save it to be used in the proposed 2-D CNN model, which is constructed from convolution, pooling, rectifier layers, the main contribution of our paper is to use 2-D system, which can be used to augment the data (images) in order to increasing the data size and to provide different viewpoints for the model, and this is used to overcome the problem of unbalanced dataset. The proposed system performance is evaluated and compared with other works. The accuracy in the training was $99.7 \%$, while in test was $91.93 \%$, precision $=92.325 \%$,recall $=92.639, \mathrm{~F} 1$ score $=91.992$, these metrics values exceeded the performance values $(88.15 \%, 85 \%, 85.53 \%$ and $85.27 \%)$ for precision, recall, F1 score respectively, for Ref[9] as described in tables. It is clear that the classifier is provided better results as compared with other approaches.

\section{REFERENCES}

[1] C. Venkastune, et al."ECG Signal Preprocessing and SVM Classifier-Based Abnormality Detection in Remote Healthcare Applications", 21693536 IEEE.

[2] A. Gacek and W. Pedrycz, "ECG Signal Processing, Classification and Interpretation A Comprehensive Framework of Computational Intelligence. London, U.K.: Springer, 2012.

[3] Pengwei Xie1, Guijin Wang1, Chenshuang Zhang1, Ming Chen1, Huazhong Yang1 ," Region Aggregation Network: Improving Convolutional Neural Network for ECG Characteristic Detection", Conference proceedings: ... Annual International Conference of the IEEE Engineering in Medicine and Biology Society. IEEE Engineering in Medicine and Biology Society,pp.2559 2562 , , 2018

[4] W. Jiang and S. G. Kong, "Block-based neural networks for personalized ECG signal classification," IEEE Transactions on Neural Networks, Vol. 18, No. 6, pp. $17501761,2007$.

[5] O. T. Inan, L. Giovangrandi, and G. T. Kovacs, "Robust neuralnetwork based classification of premature ventricular contractions using wavelet transform and timing interval features," IEEE transactions on Biomedical Engineering, Vol. 53, No. 12, pp. $25072515,2006$.

[6] Kan Luo, Jianqing Li, Zhigang Wang and and Alfred Cuschieri3, "Patient Specific Deep Architectural Model for ECG Classification", Hindawi Journal of Healthcare Engineering Volume 2017, Article ID 4108720, 13 pages https://doi.org/10.1155/2017/4108720. ,2017.

[7] Zhaohan Xiong , Martin K. Stilesand, Jichao Zhao1, " Robust ECG Signal Classification for Detection of Atrial Fibrillation Using a Novel Neural Network", Computing in Cardiology 2017; VOL 44, 2017.

[8] Mr. Ankit Sanghvi and Prof. Sachin M. Bojewar, "ECG Signal Classification Using Hidden Markov Model and Artificial Neural Network", International Journal of Engineering Research \& Technology (IJERT) Vol. 3 , No. 2, 2014.

[9] Stalin Subbiah, Rajkumar Patro and Subbuthai P., "Feature Extraction and Classification for ECG Signal Processing based on Artificial Neural Network and Machine Learning Approach", International Conference on Inter Disciplinary Research in Engineering and Technology [ICIDRET], 2015.

[10] Mohammad Kachuee, Shayan Fazeli and Majid Sarrafzadeh, "ECG Heartbeat Classification: A Deep Transferable Representation", arXiv: $1805.00794 \mathrm{v} 2$ [cs.CY] $12,2018$.

[11] Tae Joon Jun, Hoang Minh Nguyen, Daeyoun Kang, Dohyeun Kim, Daeyoung Kim and Young Hak Kim, "ECG arrhythmia classification using a 2 D convolutional neural network", arXiv:1804.06812v1 [cs.CV] 18, 2018.

[12] Kyungna Kim , "Arrhythmia Classification in Multi Channel ECG Signals Using Deep Neural Networks " master thesis, Electrical Engineering and Computer Sciences University of California at Berkeley Technical Report No. UCB/EECS-2018-80 http://www2.eecs.berkeley.edu/Pubs/TechRpts/2018/EECS-2018-80.html May 19, 2018.

[13] H. Y. Lin, S. Y. Liang, Y. L. Ho, Y. H. Lin, and H. P. Ma, "Discrete wavelet transform based noise removal and feature extraction for ECG signals," IRBM, vol. 35, no. 6, pp. 351 361, 2014. 
[14] L. Zaor alek, J. Plato s andV. Sn a sel, " Patient adapted and inter patient ECG classification using neural network and gradient boosting, CTU FTS, 2018.

[15] Iryna Mykoliuk, Daniel Jancarczyk, Mikolaj Karpinski and Viktor Kifer, "Machine Learning Methods in Electrocardiography Classification", CEUR WS.org/VOL 2300,ACIT 2018, June 1 3, 2018.

[16] Rajendra Acharya, Hamido Fujita dShu Lih Oh a, Yuki Hagiwar and Muhammad Adam , "Deep convolutional neural network for the automated detection and diagnosis of seizure using EEG signals", ELESIVER, https://www.sciencedirect.com/science/journal/ 00104825, Vol.100, p.p. 270 278. 1 September 2018. 